\title{
Enseñanza con aprendizaje móvil en educación secundaria. Percepción de la comunidad educativa
}

\author{
Teaching with mobile learning in secondary education. \\ Perception of the educational community
}

\section{Ensinar com aprendizagem móvel no ensino secundário. Percepção da comunidade educativa}

\author{
Ana Isabel Gómez-Vallecillo \\ Universidad Católica de Ávila \\ Ávila, España \\ anai.gomez@ucavila.es \\ DD ORCID: https://orcid.org/0000-0002-9907-280X \\ Diego Vergara Rodríguez \\ Universidad Católica de Ávila \\ Ávila, España \\ diego.vergara@ucavila.es \\ (D) ORCID: http://orcid.org/0000-0003-3710-4818
}

Recibido - Received - Recibido: 30/ 04 / 2021 Corregido - Revised - Revisado: 29 / 07 / 2021 Aceptado - Accepted - Aprobado: 30 / 09 / 2021

DOl: https://doi.org/10.22458/ie.v23iespecial.3514

URL: https://revistas.uned.ac.cr/index.php/innovaciones/article/view/3514

\begin{abstract}
Resumen: Este artículo difunde los resultados de una investigación evaluativa de algunos aspectos del programa de integración de tabletas digitales en el aula, desde las percepciones de una comunidad educativa concreta de Educación Secundaria tras cuatro años de implantación. Primero, se analizan cuantitativamente y con un diseño de tipo descriptivo, los cuestionarios dirigidos a 48 estudiantes y 21 familias. Seguidamente, de forma cualitativa se describen las opiniones de tres profesores y un miembro del Servicio Técnico por medio de entrevistas semiestructuradas con respecto a su satisfacción y la idoneidad del uso de la tableta como herramienta educativa. Los hallazgos ponen de manifiesto la aceptación de la comunidad educativa hacia la tableta digital, a la vez que se identifican algunos de los retos a la hora de implementar el proceso de enseñanza-aprendizaje con tabletas en la educación (tecnología informática de la escuela, competencia docente relacionadas con la enseñanza y el aprendizaje digital, retirada de libro en papel). Se concluye que el programa de implantación de las tabletas ha sido percibido como positivo, si bien existe espacio para la mejora desde el punto de vista de la conectividad y la aplicación de un modelo pedagógico cognoscitivo y colaborativo.
\end{abstract}

Palabras clave: Aprendizaje móvil, tableta digital, tecnología educacional, tecnología de la información, enseñanza secundaria.

Summary: This article disseminates the results of an evaluative investigation of some aspects of the digital tablet integration program in the classroom, based on the perceptions of a specific secondary education community after four years of implementation. First, the questionnaires addressed to 48 students and 21 families are analyzed quantitatively and with a descriptive design. Then, in a qualitative way, the opinions of three teachers and a member of the Technical Service are described through semi-structured interviews regarding their satisfaction and the suitability of using the tablet as an educational tool. The findings show the acceptance of the educational community towards the digital tablet, while identifying some of the challenges when implementing the teaching-learning process with tablets in education (school computer technology, competition teacher related to digital teaching and learning, paper book withdrawal). It is concluded that the tablet implementation program has been perceived as positive, although there is room for improvement from the point of view of connectivity and the application of a cognitive and collaborative pedagogical model.

Key Words: Mobile learning, digital tablet, educational technology, information technology, secondary education. 
Resumo: Este artigo divulga os resultados de uma pesquisa avaliativa de alguns fatores do programa de integração de tablets digitais na sala de aula, a partir das percepções de uma comunidade educacional específica do Ensino Secundário após quatro anos de implementação. Primeiro, os questionários dirigidos a 48 estudantes e 21 famílias são analisados quantitativamente e com um desenho descritivo. Em seguida, qualitativamente, as opiniões de três professores e um membro do Serviço Técnico são descritas em entrevistas semi-estruturadas sobre sua satisfação e a adequação do uso da Tablet como ferramenta educacional. Os resultados destacam a aceitação da comunidade educacional em relação à tablet digital, enquanto identificam alguns dos desafios na implementação do processo ensino-aprendizagem com tablets na educação (informática escolar, competência de ensino relacionada ao ensino e aprendizagem digital, retirada do livro impresso) e concluem que o programa de implementação da tablet tem sido visto como positivo, embora haja espaço para melhorias do ponto de vista da conectividade e da aplicação de um modelo pedagógico cognitivo e colaborativo.

Palavras-chave: Aprendizagem móvel, tablet digital, tecnologia educacional, tecnologia da informação, educação secundária.

\section{INTRODUCCIÓN}

A medida que se generaliza la adopción de las tecnologías en educación, la literatura científica abunda en trabajos que intentan demostrar el valor de incorporar los dispositivos móviles (reunidos bajo la denominación de m-learning) a la enseñanza, en virtud del potencial de las Tecnologías de la Información y Comunicación (TIC) para promover mejoras en las prácticas educativas.

El m-learning implica el uso de la tecnología móvil para permitir el aprendizaje en cualquier momento y lugar e incluye, entre otros, teléfonos móviles, tabletas y e-readers (Kraut, 2013; Fernández, Vergara, Polo \& Fernández, 2021). Estas tecnologías han eliminado las fronteras geográficas al permitir entornos de aprendizaje cooperativo (Saccol, Barbosa, Schlemmer \& Reinbard, 2010). Aunque existen dificultades para los estudiantes de todas las edades durante el aprendizaje en la práctica de la autorregulación, Sha, Looi, Chen, Seow \& Wong (2012) y Camacho (2017) indicaron que los entornos m-learning, permiten a los estudiantes aprender por sí mismos; también juegan un importante papel en la práctica del comportamiento cognitivo.

Concretamente Kowalski, Kowalski \& Gardner (2009) corroboran el aumento de la meta cognición del estudiante en el uso de tabletas digitales. Los documentos de trabajo de la UNESCO sobre el aprendizaje móvil aconsejan adoptarlo con base en la constatación de diversos beneficios (West, 2012). Sin embargo, pese a los esfuerzos y las inversiones realizadas por iniciativas públicas y privadas, no todos los estudios coinciden en su eficacia.

En el 2016 el centro escolar que ha servido de base a este estudio inició un programa de integración de un iPad por alumno, a partir de tercer curso de la Enseñanza Secundaria Obligatoria (ESO). La adopción del iPad y la implantación exitosa de este en el proceso de enseñanza-aprendizaje le ha merecido al colegio una distinción concedida por Apple a los centros que sirven de referencia en la incorporación de sus dispositivos en el aula.

Esta implementación venía precedida de infinidad de promesas sobre mejoras en el proceso de enseñanza-aprendizaje: permitir una educación más personalizada, abierta, inclusiva, en la que el alumno pudiera indagar por su cuenta y estar mucho más motivado. Pero, ¿realmente se han cumplido estas expectativas? El presente trabajo pretende conocer las percepciones individuales en la aceptación y la eficacia percibida por una comunidad educativa concreta de Educación Secundaria, por medio de una investigación evaluativa de un programa de enseñanza con tableta digital durante cuatro años.

En primer lugar, para la investigación mencionada se elaboró una revisión de la literatura con el fin de analizar las ventajas y las desventajas de la integración de tabletas digitales en el aula, así como las actitudes de la comunidad educativa hacia la enseñanza con tabletas. En una segunda fase, con un enfoque cuantitativo y cualitativo, se ha analizado cómo se ha realizado la implementación de la tableta digital 
en el centro, la opinión de los grupos de interés: estudiantado, familias, profesorado y servicio técnico y cuáles dificultades han encontrado. Por último, se ha hecho un análisis comparativo de las problemáticas detectadas y se han aportado propuestas de soluciones nacidas del debate con los distintos grupos afectados.

Los objetivos de la investigación fueron:

- Destacar las precepciones de la comunidad educativa respecto al uso de las tabletas dentro y fuera del aula, a la utilidad percibida, como dinamizadores o distractores en el aprendizaje, como apoyo al aprendizaje y los obstáculos encontrados.

- Determinar la idoneidad de la supresión del libro en papel.

- Establecer diferencias significativas entre la edad del profesorado y su satisfacción y aceptación del uso de la tableta.

- Ofrecer propuestas que permitan orientar los procesos de trabajo en el desempeño de los profesores y la efectividad del programa.

Los resultados obtenidos permitirán valorar, interpretar y juzgar los logros del programa, con el fin de aportar soluciones e identificar un modelo pedagógico apropiado para la integración de la tableta en educación. Por lo tanto, los resultados de este trabajo vienen a sumarse al acervo de experiencias de aprendizaje con tabletas.

\section{Marco teórico}

Durante la última década la mayoría de los estudios dan cuenta de la disparidad de resultados obtenidos en el uso educativo de la tableta y abarcan desde la identificación de ventajas únicas hasta el reconocimiento de su escasa relevancia en los procesos y resultados de aprendizaje. Numerosos estudios (Kim \& Frick, 2011; Lee, Lin \& Robertson, 2012; Huffman \& Hahnb, 2015; Chen \& Yan, 2016) destacan su potencial para favorecer el proceso de aprendizaje con base en el aumento de la motivación que genera el uso de una herramienta atractiva, entretenida y divertida (Furió, Seguí \& Vivó, 2015; Ciampa, 2014; Bullock, 2001). Otros trabajos han demostrado que su uso facilita la cooperación, la inclusión social, la participación y la interactividad en el aprendizaje (Camacho, 2017).

A su vez, el uso de tabletas en el aprendizaje permite multiplicar las posibilidades de trabajo en equipo, andamiaje y aprendizaje auto dirigido, al tiempo que mejora la comunicación entre profesores y estudiantes (Haßler, Major \& Hennessy, 2016). Allegra, Chifari \& Ottaviano (2001) y Wheeler, Waite \& Bromfield (2002) enfatizan que estos dispositivos estimulan la iniciativa y la creatividad en el trabajo de los estudiantes y permiten la individualización y flexibiliza tiempos y espacios. A su vez, estos beneficios fomentan la autorregulación y la personalización del aprendizaje (Camacho, 2017), lo cual crea entornos de aprendizaje en línea que permiten a los estudiantes disfrutar de un mayor acceso a la información y una gran variedad de recursos y materiales de aprendizaje (Seif, 2019).

En un nivel más profundo de aprendizaje, algunos autores sostienen incluso que el uso de estos dispositivos fomenta la adquisición de conocimientos (Lai Luo, Zhang, Huang \& Rozelle, 2007), el aprendizaje basado en la indagación y la percepción de mejora de los estudiantes en el rendimiento académico (Haßler et al., 2016; Camacho, 2017). Esto se evidencia notablemente en estudiantes desmotivados y con dificultades de aprendizaje, quienes perciben un clima más favorable en las clases con tabletas (Hammer, Göllner, Scheiter, Fauth \& Stürmer, 2021). 
Este escenario de transmisión del conocimiento en un contexto tecnológico interpela el ejercicio profesional de los docentes. Como consecuencia, las competencias del docente se han ampliado para incorporar el conocimiento necesario para la correcta integración de la tecnología en el aula. El enfoque más conocido fue el desarrollado por Mishra y Koehler (2006): el modelo TPCK (Technological Pedagogical Content Knowledge), "Conocimiento Técnico Pedagógico del Contenido". Este requiere la integración de tres tipos de conocimiento: contenido, pedagogía y tecnología, los tres componentes son la base de una enseñanza eficaz con tecnología. Por lo tanto, se refiere a las actitudes del profesorado hacia la introducción de las tabletas en la enseñanza.

Ifenthaler y Schweinbenz, (2013) indican que existe una disparidad de opiniones por parte del profesorado, tanto hacia la tecnología como hacia las expectativas de rendimiento y las condiciones que facilitan su introducción en las aulas. Al respecto, Suárez-Guerrero, Lloret-Catalá \& Mengual-Andrés (2016) en su estudio acerca de la percepción docente sobre la transformación digital del aula, señalan que pese a que los docentes conceden ciertos beneficios asociados al uso de tabletas en términos de aprendizaje basado en proyectos, competencias digitales o actividades interdisciplinares; sin embargo, "no es posible afirmar con rotundidad que exista un «impacto positivo» de su incorporación a la educación formal". (p. 82)

La valoración de los padres sobre la integración de los dispositivos del $m$-learning en el sistema educativo es generalmente positiva, incluso una vez reconocidos los riesgos y los efectos negativos de las tecnologías móviles, como son el cyber acoso o el uso inadecuado de las redes sociales (Torrecillas-Lacave, Vázquez-Barrio \& Monteagudo-Barandalla, 2017).

Las investigaciones sobre la intención conductual de los estudiantes en el uso de las tabletas con un fin académico muestran que la expectativa de rendimiento, la capacidad para hacer el aprendizaje más entretenido, estimular la curiosidad y la facilidad de búsqueda de información adicional resultan fundamentales a la hora de determinar las intenciones de comportamiento de los estudiantes (Hamzah, Yusoff, Ismail \& Yacob, 2020). En este sentido, según Yáñez-Luna y Arias-Oliva (2018) los estudiantes consideran el m-learning de gran utilidad para realizar sus actividades académicas; mientras Fuentes, Albertos \& Torrano (2019) presentan en sus resultados que la mayoría de los estudiantes perciben un aumento de rendimiento académico a partir de su empleo continuado.

Todas estas ventajas deberían conducir a una mejora del rendimiento académico y a la adquisición de competencias por parte del alumnado (Gómez-Fernández y Mediavilla, 2018). Sin embargo, también abundan los estudios que constatan efectos negativos en el uso de tabletas en la educación. Fox, Rosen \& Crawford (2009) indican que las tabletas se presentan como una distracción para los estudiantes, que además conlleva excesiva pérdida de tiempo en la búsqueda de información, lo cual resulta ser poco fidedigna. Gómez-Fernández y Mediavilla (2018) interpretan que estos inconvenientes pueden tener consecuencias negativas no solo en el desarrollo personal y social de los estudiantes, sino también en su rendimiento académico y en la adquisición de competencias.

En este sentido, el informe "Estudiantes, Ordenadores y Aprendizaje: realizando la conexión" de la Organización para la Cooperación y Desarrollo Económico (OECD, 2015) revela que incluso los países que han efectuado grandes inversiones en la digitalización de las aulas no han mostrado una mejoría notoria en sus resultados del informe PISA (Programme for International Student Assessment) en cuanto a comprensión lectora, matemáticas o ciencia.

El estudio de Jiménez-Martín \& Vilaplana (2014) sustenta el efecto negativo sobre el rendimiento académico en la nota de matemáticas como resultado de la aplicación de tabletas en la enseñanza. Los trabajos de Nguyen, Barton \& Nguyen (2015), Meyer (2016) y Dhir, Gahwaji \& Nyman (2013) evidencian que no se producen mejoras en términos de rendimiento académico, y hay quien llega incluso a reconocer que las tabletas son un impedimento propiamente dicho para el aprendizaje (Mueller y Oppenheimer, 2014). 
El programa dentro del cual se llevó a cabo la presente investigación es en el contexto de un colegio privado, bilingüe (español-inglés), con un proyecto de implantación masiva de tabletas digitales (concretamente iPads) dentro de sus aulas, desde el curso 2015-2016, con el objetivo de suprimir progresivamente los libros en papel, así como implementar la enseñanza cooperativa en todos los niveles.

\section{MATERIALES Y MÉTODOS}

La metodología aplicada en este estudio es la investigación evaluativa, definida por Mateo (2000) como "un proceso de recogida de información orientado a la emisión de juicios de mérito o de valor respecto de algún sujeto, objeto o intervención" (p. 21). Esta modalidad de investigación está orientada a la toma de decisiones y "a determinar la eficacia de organizaciones y programas educativos" (Sandín, 2003, p. 173). La perspectiva evaluativa adoptada es la evaluación de un programa o producto, que "nos proporciona información para tomar decisiones de reciclaje con el fin de aceptar, rectificar o abandonar el programa." (Bausela, 2003, p. 367).

En el diseño de la investigación se ha optado por combinar el enfoque cuantitativo y el cualitativo. En un primer momento y desde la perspectiva cuantitativa, la investigación se caracteriza como exploratoria y descriptiva. Para ello se utilizó un cuestionario al alumnado (Tabla 1) y a sus familias (Tabla 2), para medir las variables no cuantificables: (i) el uso que hacen los discentes de la tableta dentro y fuera del aula, (ii) la tableta como distractor, las opiniones sobre las aplicaciones propuestas y preferencias frente al libro en papel, (iii) el grado de satisfacción en el uso de la tableta y (iv) el uso que hacen las familias de las TIC como soporte de comunicación con el centro escolar.

TABLA 1

Cuestionario para los estudiantes

\begin{tabular}{lccc}
\multicolumn{1}{c}{ Preguntas } & & \multicolumn{2}{c}{ Respuestas posibles } \\
\hline Q1. ¿Te resulta complicado manejar un iPad? & Sí & A veces & No \\
\hline Q2. ¿Te distraes con frecuencia usando el iPad? & Sí & A veces & No \\
\hline Q3. ¿En qué inviertes más horas en el iPad? & Jugar & Ver vídeos/series & Estudiar \\
\hline Q4. ¿En dónde usas más el iPad: en el aula o en casa? & Aula & Casa & A veces \\
\hline Q5. ¿Utilizas el iPad en casa para un uso académico? & Sí & Casi siempre & Digital \\
\hline Q6. ¿En qué formato prefieres leer un libro? & Físico & No \\
\hline $\begin{array}{l}\text { Q7. Si pudieras volver a recibir clase con libros en formato papel, } \\
\text { ¿lo preferirías? }\end{array}$ & Sí & Tal vez & Casi \\
\hline Q8. ¿Te parecen útiles las aplicaciones proporcionadas por el colegio? & Sí & La mayoría & ninguna \\
\hline
\end{tabular}

Fuente: elaboración propia. 
TABLA 2

Cuestionario para las familias

\begin{tabular}{|c|c|c|c|c|c|}
\hline Preguntas & & Res & lestas po & & \\
\hline $\begin{array}{l}\text { Q9. ¿Está satisfecho con la implantación del iPad en el } \\
\text { colegio? }\end{array}$ & 1 & 2 & 3 & 4 & 5 \\
\hline $\begin{array}{l}\text { Q10. ¿Considera beneficioso el uso de las nuevas tecnolo- } \\
\text { gías en educación? }\end{array}$ & 1 & 2 & 3 & 4 & 5 \\
\hline $\begin{array}{l}\text { Q11. Mientras estaba usted en el colegio, si hubiese tenido } \\
\text { la oportunidad, ¿le hubiese gustado hacer uso de las nuevas } \\
\text { tecnologías en el ámbito académico? }\end{array}$ & Sí & No & & & \\
\hline $\begin{array}{l}\text { Q12. ¿Considera que el iPad puede ocasionar distracciones } \\
\text { en su hijo/a? }\end{array}$ & Sí & No & & & \\
\hline $\begin{array}{l}\text { Q13. Personalmente, ¿prefiere los libros en formato papel o } \\
\text { digital? }\end{array}$ & Papel & Digital & Ambos & & \\
\hline $\begin{array}{l}\text { Q14. ¿Qué formato prefiere para las notificaciones, informes, } \\
\text { circulares y boletines de notas? }\end{array}$ & Papel & Digital & Ambos & & \\
\hline $\begin{array}{l}\text { Q15. Como padre/madre, zaccede con frecuencia a la plata- } \\
\text { forma online "Alexia"? }\end{array}$ & $\begin{array}{c}\text { Con } \\
\text { frecuencia }\end{array}$ & $A$ veces & Nunca & & \\
\hline
\end{tabular}

Nota: Valore las preguntas Q9 y Q 10 en una escala del 1 al 5, siendo el 1. Totalmente en desacuerdo y el 5. Totalmente de acuerdo.

Fuente: elaboración propia.

El cuestionario se completó por los estudiantes dentro del aula. Los profesores estaban presentes para controlar tanto el proceso de respuesta como la comprensión de los sujetos entrevistados, lo que proporciona un impacto mayor en la calidad de las respuestas.

En un segundo momento, y desde la perspectiva cualitativa, la herramienta de recogida de información fue la entrevista semiestructurada, exploratoria y correlacional de preguntas abiertas a tres profesores (Bisquerra, 2016). Estas tuvieron una duración aproximada de 30 minutos y se realizaron en un área predeterminada en el propio centro escolar. Las entrevistas sirvieron para medir el grado de relación existente entre la variable de edad del profesorado y la aceptación de la introducción de la tableta en el aula, así como documentar sus experiencias sobre el rendimiento percibido y los obstáculos que han encontrado en el proceso de transformación de enseñanza con tabletas. Por último, tuvo lugar la entrevista semiestructurada al único miembro de Soporte Técnico, que arrojó luz cualificada sobre otro punto de vista que a menudo se obvia y es la opinión de los expertos en tecnología.

Para análisis de datos en el enfoque cuantitativo se realizó un cálculo de porcentajes a partir de los datos obtenidos de las muestras. Para el análisis de los datos cualitativos, se tomaron notas de las entrevistas, se redujeron posteriormente los datos no estructurados y se codificaron de forma abierta sobre las categorías de: (1) valoración general del iPad en el aula como herramienta educativa y (2) dificultades experimentadas en la implantación de este.

\section{Población de estudio}

Discentes: la muestra, de carácter no probabilístico, intencional y por conveniencia (Bisquerra, 2016), se compone de los 48 estudiantes de segundo de bachillerato de un colegio privado de un área urbana. Estos estudiantes llevaban cuatro años usando esta tecnología (iPad) antes de la encuesta. Para fomentar la motivación, se integró este estudio dentro de un proyecto sobre marketing relacional que comprendía la nota del segundo trimestre en la asignatura de Economía de la Empresa. 
Familias: se suministró un cuestionario online usando Google Forms dirigido a las familias de los estudiantes y se obtuvieron 21 encuestas. La muestra fue de carácter no probabilístico, intencional y por conveniencia.

Profesorado: la muestra, de carácter no probabilístico, intencional y por conveniencia, la forman tres profesores con características diferenciales. Para medir el grado de relación existente sobre la variable de la edad en la aceptación de la tableta digital, se eligió a un profesor de biología en bachillerato mayor de sesenta años (profesor A), un profesor de informática para secundaria y bachillerato, menor de cuarenta años y miembro del departamento de innovación (profesor B) y una profesora de idiomas menor de treinta años (profesor C).

Servicio técnico: casi todos los problemas relacionados con los dispositivos móviles pasan en algún momento por el departamento técnico y el departamento está compuesto por una sola persona que se encarga casi en exclusiva del entorno de red, el contacto directo con Apple, las configuraciones de iPad y el mantenimiento de estos.

\section{DISCUSIÓN DE RESULTADOS}

En primer lugar, se presentan los resultados por grupos de encuestados. Se empezó por el centro escolar: estudiantes y profesorado; y se finalizó con familias y el departamento técnico.

\section{Resultados del cuestionario al alumnado}

Según se observa en la Tabla 3, a la pregunta acerca de la facilidad de uso de la tableta, un 16,7\% considera que en ocasiones es complicado. Por lo tanto, partimos de la base de que los estudiantes no presentan dificultades en el manejo de la tecnología. Las distracciones en el uso de la tableta resultan ser muy frecuentes y el 37,5\% es capaz de hacer uso del dispositivo sin distraerse con otras aplicaciones. En cuanto al uso que los discentes efectúan del iPad el 58,3\% reconoce utilizarlo con fines de entretenimiento y un $41,7 \%$ hace uso de la tableta con fines académicos.

TABLA 3

Respuestas a las preguntas Q1, Q2 y Q3. Facilidad de uso y distracciones al usar el iPad

\begin{tabular}{lccc}
\hline ¿Te resulta complicado manejar un iPad? & Sí & A veces & No \\
\hline ¿Te distraes con frecuencia usando el iPad? & $0 \%$ & $16,70 \%$ & $83,30 \%$ \\
\hline ¿En qué inviertes más horas en el iPad? & $29,20 \%$ & $33,30 \%$ & $37,50 \%$ \\
\hline
\end{tabular}

Fuente: elaboración propia.

Con respecto a la utilización de la tableta, la mayoría de los estudiantes hace uso de ella en el aula, tal y como muestra la Tabla 4; mientras el $35,4 \%$ lo hace en casa. Las respuestas al uso académico en el hogar resultan congruentes con las respuestas dadas al uso real del iPad. En este caso, el porcentaje de estudiantes que admite usar la tableta para jugar, coincide con el porcentaje de estudiantes que nunca hacen uso de ella para las tareas de clase, frente a más de la mitad que reconoce utilizarla en el hogar con distintos propósitos. El dato de que un $35,4 \%$ de los estudiantes solo a veces o nunca usan el iPad en casa para uso académico, nos revela que es una herramienta pensada para el estudio pero que los discentes no la perciben como tal. 
TABLA 4

Respuestas a las preguntas Q4 y Q5. Uso académico en el hogar

\begin{tabular}{lccccc}
\hline ¿En dónde usas más el iPad: en el aula o en casa? & Aula & Casa & & \\
& $65 \%$ & $35 \%$ & & \\
\hline ¿Utilizas el iPad en casa para un uso académico? & Sí & Casi siempre & A veces & Nunca \\
& $31,30 \%$ & $33,30 \%$ & $27,10 \%$ & $8,30 \%$ \\
\hline
\end{tabular}

Fuente: elaboración propia.

En la comparativa sobre la preferencia entre la lectura de libros en el iPad o papel, los estudiantes muestran preferencia del $89,6 \%$ por la lectura de libros en formato en papel tradicional (Tabla 5). Un 20,8\% de los encuestados no desearía volver a dar clase con los libros en formato papel. Dato muy esclarecedor, que indica de nuevo la preferencia del estudiantado por el libro en papel, si bien un 27,1\% parece sopesar otras razones y no está seguro de querer deshacerse de los libros digitales. El iPad además de usarse como libro electrónico es soporte de aplicaciones educativas. A la pregunta de si les parecen útiles las aplicaciones proporcionadas por el colegio, un 4,2\% responde con rotundidad que sí y un $50 \%$ apunta que la mayoría sí; por lo tanto, un $45,8 \%$ de la población del estudio considera que casi ninguna o ninguna aplicación de las proporcionadas por el colegio es útil.

TABLA 5

Respuestas a las preguntas Q6, Q7 y Q8. Formato preferido para la lectura de libros y valoración de las aplicaciones educativas

\begin{tabular}{lrrrr}
\hline ¿En qué formato prefieres leer un libro? & & Físico & & Digital \\
& & $89,6 \%$ & $10,4 \%$ \\
\hline Si pudieras volver a dar clase con libros en formato papel, ¿lo preferirías? & Sí & Tal vez & No \\
\hline ¿Te parecen útiles las aplicaciones proporcionadas por el colegio? & Sí & La mayoría & Casi ninguna & No \\
\hline & $4,2 \%$ & $50 \%$ & $37,5 \%$ & $8,3 \%$ \\
\hline
\end{tabular}

Fuente: elaboración propia.

\section{Resultados de las encuestas al profesorado}

La valoración general del profesor de informática (profesor B) es muy positiva y resalta que el iPad es una herramienta que le ha permitido motivar mucho a sus estudiantes, por medio de la cual utiliza infinidad de aplicaciones de gran interés para la materia de estudio; además de permitirle desarrollar proyectos como competiciones de cortos cinematográficos o programar robots.

Por su parte, el profesor de biología (profesor A) es ligeramente favorable, considera que los estudiantes pierden el hábito de la escritura manual y se descentran con mayor facilidad, pero admite que es una herramienta poderosa, en especial para la búsqueda de información a la que hay que adaptarse tarde o temprano. La profesora de idiomas (profesor C) valoró como positiva la implantación del iPad; pues le permite buscar material audiovisual en inglés rápida y cómodamente, facilita a los estudiantes grabarse y detectar fallos en la pronunciación y preparar exposiciones sobre la marcha, aunque las posibilidades de distracción son también mayores.

En cuanto a las preocupaciones y las dificultades experimentadas por los profesores en la implantación del iPad en el aula, los resultados del análisis revelaron tres áreas destacadas que incluían: puntos de vista sobre la tecnología, sobre su desempeño profesional, y sobre el uso de las tabletas por los 
estudiantes. En primer lugar, desde el punto de vista tecnológico, los docentes apuntaron: (i) disrupciones en las sesiones por fallos técnicos con el wifi o los iPads, que afectan al desarrollo de las clases; (ii) escasez de funcionalidades y capacidad de los iPads proporcionados al profesorado; (iii) dependencia tecnológica en las limitaciones en la red de internet; y (iv) dificultad a la hora de valorar el interés de muchas aplicaciones que no permiten evaluar sus funcionalidades sino previo pago.

En segundo lugar, desde el punto de vista profesional, señalaron: (i) carencias en la formación tecnológica; (ii) excesiva carga en la preparación de contenidos y materiales académicos, que han de hacerse a expensas del tiempo libre y voluntad del profesor; (iii) duplicación de soportes en papel y digital para los trámites administrativos; (iv) aumento de la carga de trabajo por parte de la dirección del centro, una vez se ha comprobado que la tableta facilita y agiliza ciertas tareas; (v) carencia de unificación de criterios en el uso de la tableta a nivel institucional, y (vi) excesiva inversión de tiempo para conocer las funcionalidades de la herramienta.

Finalmente, acerca del uso de las tabletas por los estudiantes, los profesores indicaron: (i) falta de formación y criterios comunes frente a robos, averías o desperfectos debido a conductas inadecuadas; (ii) utilización de la tableta para usos inapropiados, como grabar a los compañeros o al profesorado y compartirlo en redes sociales; (iii) envío de archivos no académicos entre estudiantes; (iv) distracciones con juegos; (v) pérdida del hábito de tomar apuntes o consultar fuentes de información fidedignas, y (vi) posibilidad de ocultar muchos comportamientos inapropiados.

\section{Resultados del cuestionario a las familias}

En general, las familias consideran beneficioso el uso de las TIC en la educación, un 71,4\% les dan la puntuación máxima (4 o 5); mientras un $28,5 \%$ de las familias valora menos las bondades de las nuevas tecnologías en el aula (Figura 1). Sobre la satisfacción de la implantación de iPads en las aulas el 61,9\% de las familias responde estar satisfecha (puntuación 4 o 5), y el 38,1\% no le da al dispositivo una valoración alta (1 a 3) y perciben desventajas en su implantación.

Figura 1. Distribución sobre la satisfacción de la implantación de iPads y opinión de las familias sobre las TIC.

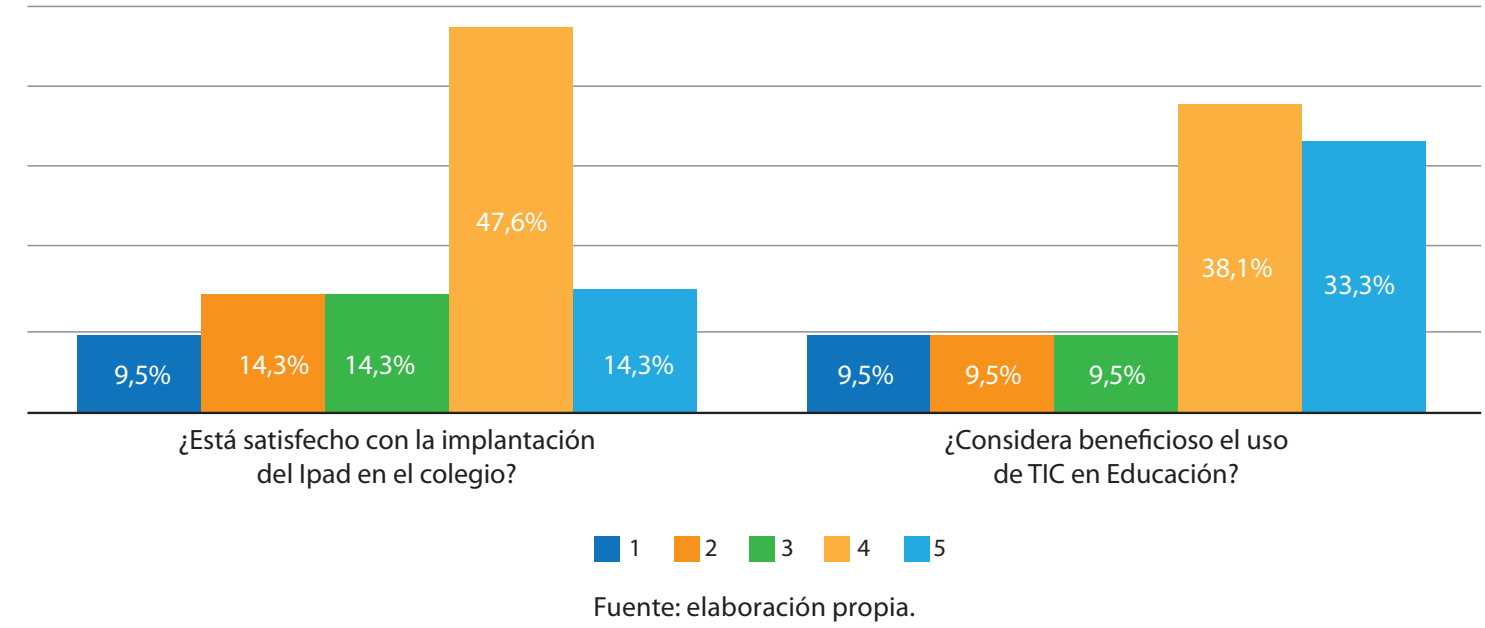


En la Tabla 6 se observa que existe un volumen elevado de familias que valora las TIC en la enseñanza y habría deseado hacer uso de ellas en su propia formación académica, pero también se nota que un $23,8 \%$ de los padres que son resistentes; pues respondieron directamente "no" a la pregunta formulada. También se observa que las familias no son ajenas a las desventajas de las tabletas y un $66,7 \%$ considera que puede provocar distracciones en sus hijos.

TABLA 6

Respuestas a las preguntas Q11 y Q12. TIC y el iPad como fuente de distracción.

\begin{tabular}{lcc} 
& Sí & No \\
$\begin{array}{l}\text { Mientras estaba usted en el colegio, si hubiese tenido la oportunidad, ¿le hubiese gustado } \\
\text { hacer uso de las nuevas tecnologías en el ámbito académico? }\end{array}$ & 76,2 & $23,8 \%$ \\
¿Considera que el iPad puede ocasionar distracciones en su hijo/a? & 66,7 & $33,3 \%$ \\
\hline
\end{tabular}

Fuente: elaboración propia.

El $0 \%$ de los padres considera que prefiere únicamente los libros en formato digital; algo más de la mitad de los encuestados los prefiere en papel y el $47,6 \%$ prefiere tener ambos. El $71,4 \%$ de las familias prefieren el formato papel para las notificaciones, informes, circulares y boletines de notas (Tabla 7).

TABLA 7

Respuestas a las preguntas Q13 y Q14. Preferencias de formato

\begin{tabular}{|c|c|c|c|}
\hline & Papel & Digital & Ambos \\
\hline Personalmente, ¿prefiere los libros en formato papel o digital? & 52,4 & $0 \%$ & 47,6 \\
\hline $\begin{array}{l}\text { ¿Qué formato prefiere para las notificaciones, informes, circulares y boletines de } \\
\text { notas? }\end{array}$ & $71,4 \%$ & $28,6 \%$ & $0 \%$ \\
\hline
\end{tabular}

Fuente: elaboración propia.

Una de las ventajas que se apunta en el uso de las nuevas tecnologías como favorecedora de la comunicación del Colegio con las familias recibe un criterio de realidad (Tabla 8). Un 0\% de los encuestados accede con frecuencia a la plataforma Alexia (plataforma online usada como Cuaderno del Profesor para la gestión de las clases y la comunicación con las familias), y un 52,4\% no accede nunca.

TABLA 8

Respuestas a la pregunta Q15. Comunicación con las familias

\begin{tabular}{cccc}
\hline ¿Con qué asiduidad accede a la plataforma online "Alexia"? & Con frecuencia & A veces & Nunca \\
\hline
\end{tabular}

Fuente: elaboración propia.

\section{Resultados de la entrevista con el departamento técnico}

La percepción global del técnico informático es que la implantación del iPad es muy necesaria. En su opinión: "Los obstáculos tienen su origen mayoritariamente en el insuficiente ancho de banda para dar acceso a todos los dispositivos a la vez junto con la escasez de routers para las aulas". Otra dificultad que detecta es el hecho de que sean las familias las propietarias de los dispositivos, pues de él derivan los problemas de privacidad, acceso a páginas inapropiadas para su edad; pues, "si bien existe un perfil de control en el colegio, los estudiantes han descubierto cómo eliminarlo". 
A lo anterior expuesto, se añaden retrasos en la adaptación a los formatos digitales de las editoriales, así como en la gestión de las licencias y la entrega en tiempo y forma de los códigos de acceso al material digital. Asimismo, señala que "la falta de actualización del software hace incompatibles los robots de robótica, de Spheros y los drones, que son muy antiguos para trabajar con la tableta". Sin embargo, según apunta el entrevistado "una vez se consiga aumentar el ancho de banda y se solvente el problema de la propiedad del iPad, la utilidad de este dispositivo con fines académicos será extraordinaria."

\section{SÍNTESIS Y REFLEXIONES FINALES}

El primer objetivo de este trabajo ha sido analizar las percepciones de los distintos grupos de interés de un centro de enseñanza secundaria al implantar el iPad por alumno, así como los obstáculos que impiden la integración académica satisfactoria del dispositivo en el aula. Aunque los resultados sugieren una amplia aceptación sobre el programa de implantación de la tableta en las aulas, también se han detectado áreas de mejora: uso inapropiado que el estudiantado hace del iPad, escasa comunicación de las familias con el centro a través de la plataforma Alexia, escasa utilidad percibida de las aplicaciones usadas en el aula y carencias en el desarrollo del profesorado de acuerdo con el modelo TPCK.

En relación con el uso inapropiado del iPad, el Marco Europeo de Competencia Digital Docente, elaborado por la Comisión Europea en 2017, identifica 21 competencias digitales divididas en cinco secciones clave. Una de ellas describe el desarrollo de la competencia digital de los estudiantes en cinco áreas: información y alfabetización mediática, comunicación, creación de contenido, uso responsable y solución de problemas. Tener las habilidades descritas anteriormente juega un papel central en la vida escolar y futuro laboral de los estudiantes. Por eso, la formación del alumnado en estas cinco áreas favorecería la prevención de conductas inapropiadas y el apoyo a procesos de aprendizaje en línea.

Otro de los puntos de mejora es la comunicación con las familias a través del iPad. Es vital que los padres aprendan a desenvolverse en los entornos digitales, con el fin de obtener el máximo provecho de las tecnologías por medio de un plan de adaptación a los dispositivos y las plataformas usadas por el colegio. Con ello además se conseguiría vencer muchos de los recelos y reservas en el uso de esta tecnología que manifestaron algunos progenitores en la encuesta, cuando declaran su insatisfacción con la integración del iPad.

En cuanto a la utilidad percibida de las aplicaciones utilizadas en el aula, los estudiantes se dividen casi al $50 \%$ entre los que valoran en el iPad una herramienta positiva para su desempeño académico y los que no. Todos estos datos apuntan a que la tableta no es la herramienta didáctica única ni la preferida por los discentes, sino que convive con otros soportes analógicos y digitales.

Los resultados de la encuesta evidencian el deficiente desarrollo profesional tecnológico del profesorado, la familiarización con el dispositivo, la falta de materiales adaptativos y la carga de trabajo asociada a la preparación de materiales. Esto coincide con estudios que apuntan como obstáculo a la introducción de las tabletas en el aula a la formación deficiente de los profesores en competencia digital, la falta de comprensión acerca del potencial y utilidad de la tableta en el aprendizaje y el modelo pedagógico utilizado.

Es evidente que la labor de los docentes se desarrolla en escenarios cada vez más complejos y multimodales, los cuales exigen la combinación de conocimientos didácticos, curriculares y tecnológicos para implementar prácticas docentes significativas con TIC, de acuerdo con el modelo TPCK. En ese sentido, se hace imprescindible una labor institucional planeada y estructurada de apoyo continuo al profesorado, así como la puesta en común de buenas prácticas educativas que utilizan dichas tecnologías de forma constructiva para enseñar los contenidos. 
El fortalecimiento en número de personal en el departamento técnico, la formación, la alianza con los proveedores del dispositivo y la búsqueda de foros de cooperación con otros centros en los cuales esté implantado resultan vitales. Ayudará a adelantarse y solucionar con prontitud problemas ocasionados por el mal uso que hacen los estudiantes de los dispositivos.

El segundo objetivo era determinar la idoneidad de la supresión del libro en papel, que es un objetivo del colegio. Los resultados del estudio arrojan que una mayoría del estudiantado prefiere leer los libros en papel y le gustaría volver al libro físico. Asimismo, ninguna de las familias prefiere tener únicamente el libro digital. Estos datos coinciden con los resultados de otros estudios, como el de Gómez (2019), elaborado a partir de 400 entrevistas a docentes y un millar de padres, que destaca la preferencia de los libros en papel sobre las tabletas, más acusado incluso entre los progenitores, reconociendo el valor del libro como una herramienta que permite estructurar y sistematizar los contenidos.

A la vista de los anteriores resultados, sería conveniente revisar colectivamente las políticas de retirada de libros de texto en papel, acciones que tal vez obedecen a intereses que nada tienen que ver con la mejora de la calidad de la enseñanza. Por fortuna, hoy muchas editoriales ofrecen el libro físico junto con el digital en un mismo paquete, de modo que los estudiantes podrían usar el libro en papel para el estudio en casa de contenidos complejos y el virtual para el seguimiento de las clases, consultas rápidas o búsqueda de información. Con estas medidas, además, atenderíamos los problemas asociados al peso de la mochila, el deterioro visual y los dolores de cabeza, cuestiones de salud prioritarias para las familias.

En cuanto al tercer objetivo, no se han encontrado diferencias significativas entre la edad del profesorado y su satisfacción y aceptación del uso de la tableta. Los tres profesores encuestados coinciden en el valor positivo de su introducción y las dificultades asociadas.

El cuarto objetivo era ofrecer propuestas que permitan orientar los procesos de trabajo en el desempeño de los profesores y la efectividad del programa. Como se ha podido revelar en este trabajo, proporcionar un dispositivo digital no garantiza que los estudiantes lo utilicen satisfactoriamente en su aprendizaje. En ese sentido, y en vista de los resultados de este estudio, se constata la necesidad de un enfoque pedagógico de aprendizaje cognoscitivo y cooperativo. El enfoque metodológico cognoscitivo pone el foco en el alumno y sus necesidades, donde el profesorado asume la tarea de dinamizar y orientar el aprendizaje y ayuda a los estudiantes a identificar sus propias necesidades de aprendizaje por medio de tareas de reflexión y autoevaluación, enseñándoles a emplear estrategias de aprendizaje eficaces y proporcionándoles orientación para organizar su aprendizaje.

El modelo didáctico cooperativo resulta ser el más apropiado para crear experiencias educativas con tabletas: trabajo por proyectos multidisciplinar con resultados de aprendizaje que puedan compartirse con la comunidad, hacia la presentación de la información de manera creativa y como herramienta de comunicación y participación activa de los estudiantes.

Los resultados aquí presentados no se pueden generalizar a otros centros educativos, dado el número tan pequeño de muestra estudiada. Asimismo, aspectos como el diseño de sistemas de evaluación adaptados a la nueva metodología didáctica con tableta no ha sido abordado en este estudio. Estas limitaciones invitan a futuras investigaciones en esta dirección. 
Allegra, M., Chifari, A. \& Ottaviano, S. (2001). ICT to train students towards creative thinking. Educational Technology \& Society, 4(2), 48-53.

Bausela, E. (2003). Metodología de la investigación evaluativa: modelo CIPP. Revista complutense de educación, 14(2), 361-376.

Bisquerra, R. (2016) (Coord.). Metodología de la investigación educativa. Madrid, España: Editorial Arco/ Libros-La Muralla.

Bullock, J. (2001). Evaluating the impact of using ICT upon student motivation and attainment in English. Recuperado de https://bit.ly/32Ce35F

Camacho, M. (2017). Tablets en educación. Hacia un aprendizaje basado en competencias. Samsung Smart School 2016-17. Recuperado de https://bit.ly/3nbwNCC

Chen, Q., \& Yan, Z. (2016). Does multitasking with mobile phones affect learning? A review. Computers in Human Behavior, 54, 34-42. http://dx.doi.org/10.1016/j.chb.2015.07.047

Ciampa, K. (2014). Learning in a mobile age: an investigation of student motivation: Learning in a mobile age. Journal of Computer Assisted Learning, 30(1), 82-96. http://doi:10.1111/jcal.12036

Dhir, A., Gahwaji, N. M., \& Nyman, G. (2013). The Role of the iPad in the Hands of the Learner. Journal of Universal Computer Science, 19(5), 706-727.

Fernández, P., Vergara, D., Polo, J., \& Fernández, M. L. (2021). Revisión de la implantación del m-learning como método de aprendizaje en España en los niveles educativos de primaria y secundaria. Revista Educativa Hekademos, 30, 60-71.

Fox, A. B., Rosen J., \& Crawford M. (2009). Distractions, distractions: does instant messaging affect college students' performance on a concurrent reading comprehension task? Cyberpsychol. Behav, 12(1), 51-3. http://doi: 10.1089/cpb.2008.0107

Fuentes, J. L., Albertos, J. E., \& Torrano, F. (2019). Towards the mobile-learning in the school: Analysis of critical factors on the use of tablets in Spanish schools. Education in the Knowledge Society, 20, 1-17.

Furió, D., Juan, M. C., Seguí, I., \& Vivó, R. (2015). Mobile learning vs. traditional classroom lessons: a comparative study. Journal of Computer Assisted Learning, 31(3), 189-201.

Gómez, R. (2019). Estudio sobre el papel y valor de los contenidos educativos en las aulas. Grupo Análisis e Investigación. Recuperado de http://www.analisiseinvestigacion.com/

Gómez-Fernández, N., \& Mediavilla, M. (2018). Do information and communication technologies (ICT) improve educational outcomes? Evidence for Spain in PISA 2015. Recuperado de https://bit. ly/3n5VCQj

Hammer, M., Göllner, R., Scheiter, K., Fauth, B., \& Stürmer, K. (2021). For whom do tablets make a difference? Examining student profiles and perceptions of instruction with tablets. Computers \& Education, 166, 104147.

Hamzah, W. W., Yusoff, M., Ismail, I., \& Yacob, A. (2020). The Behavioural Intentions of Secondary School Students to Use Tablet as a Mobile Learning Device. International Journal of Interactive Mobile Technologies, 14(13), 161-171.

Haßler, B., Major, L., \& Hennessy, S. (2016). Tablet use in schools: A critical review of the evidence for learning outcomes. Journal of Computer Assisted Learning, 32(2), 139-156.

Huffman, W. B., \& Hahn, S. (2015). Cognitive Principles in Mobile Learning Applications. Psychology, 6(04), 456-463. 
Ifenthaler, D., \& Schweinbenz, V. (2013). The acceptance of Tablet-PCs in classroom instruction: The teachers' perspectives. Computers in human behavior, 29(3), 525-534.

Jiménez-Martín, S., \& Vilaplana, C. (2014). Evaluación del Programa Escuela 2.0 a partir de los resultados en Matemáticas de PISA 2012. Recuperado de https://bit.ly/3tH8Kh7

Kim, K.-J., \& Frick, T. W. (2011). Changes in student motivation during online learning. Journal of Educational Computing Research, 44(1), 1-23.

Kowalski, S. E., Kowalski, F. V., \& Gardner, T. Q. (2009). Lessons learned when gathering real-time formative assessment in the university classroom using tablet PCs. In 2009 39th IEEE Frontiers in Education Conference, 1-5.

Kraut, R. (Ed.). (2013). Policy guidelines for mobile learning. Unesco.

Lai, F., Luo, R., Zhang, L., Huang, X. \& Rozelle, S. (2015). Does computer-assisted learning improve learning outcomes? Evidence from a randomized experiment in migrant schools in Beijing. Economics of Education Review, 47, 34-48. http://dx.doi.org/10.1016/j.econedurev.2015.03.005

Lee, J., Lin, L., \& Robertson, T. (2012). The impact of media multitasking on learning. Learning, Media and Technology, 37, 94-104. http://doi:10.1080/17439884.2010.537664

Mateo, J. (2000). La investigación educativa, su práctica y otras metáforas. Barcelona, España: ICE-UB/ Horsori.

Meyer, B. (2016). Mobile devices and spatial enactments of learning: IPads in lower secondary schools. Proceedings of the 12th International Conference on Mobile Learning, 3-10. Recuperado de https:// bit.ly/3aw28dV

Mishra, P., \& Koehler, M. J. (2006). Technological pedagogical content knowledge: A framework for teacher knowledge. Teachers College Record, 108(6), 1017-1054.

https://doi:10.1111/j.1467-9620.2006.00684.x.

Moreira, F., Ferreira, M. J., Santos, C. P., \& Durão, N. (2017). Evolution and use of mobile devices in higher education: A case study in Portuguese higher education institutions between 2009/2010 and 2014/2015. Telematics and Informatics, 34 (6), 838-852. https://doi.org /10.1016/j.tele.2016.08.010

Mueller, P. A., \& Oppenheimer, D. M. (2014). The pen is mightier than the keyboard: Advantages of longhand over laptop note taking. Psychological science, 25(6), 1159-1168.

Nguyen, L., Barton, S. M., \& Nguyen, L. T. (2015). iPads in higher education-Hype and hope. British Journal of Educational Technology, 46(1), 190-203. http://doi:10.1111/bjet.12137

OECD (2015), Students, Computers and Learning: Making the Connection, PISA, OECD Publishing. http://dx.doi.org/10.1787/9789264239555-en

Saccol, A., Barbosa, J. I. V., Schlemmer, E., \& Rienhard, N. (2010). Corporate m-learning: applications and challenges. In Guy (Ed.) Mobile Learning: Pilot Projects and Initiatives (pp. 215-242). California, USA: Information Science Press.

Sandín, M. P. (2003). Investigación cualitativa en educación. Fundamentos y tradiciones. Madrid, España: McGraw Hill.

Seif, J. (2019). Mobile Technologies and Mobile Learning: How has the use of tablets innovated learning within Secondary School classrooms? Technology and the Curriculum: Summer 2019. Recuperado de https://bit.ly/3tDpVAk

Sha, L., Looi, C. K., Chen, W., Seow, P., \& Wong, L. H. (2012). Recognizing and measuring self-regulated learning in a mobile learning environment. Computers in Human Behavior, 28(2), 718-728. 
Suárez-Guerrero, C., Lloret-Catalá, C., \& Mengual-Andrés, S. (2016). Percepción docente sobre la transformación digital del aula a través de tabletas: un estudio en el contexto español. Comunicar: Revista Científica de Comunicación y Educación, 24(49), 81-89.

Torrecillas-Lacave, T., Vázquez-Barrio, T., \& Monteagudo-Barandalla, L. (2017). Percepción de los padres sobre el empoderamiento digital de las familias en hogares hiperconectados. El profesional de la información (EPI), 26(1), 97-104. https://doi.org/10.3145/epi.2017.ene.10

West, M. (2012). Activando el aprendizaje móvil: Temas globales. Serie de documentos de trabajo de la UNESCO sobre el aprendizaje móvil. Recuperado de https://bit.ly/3tHxWnM

Wheeler, S., Waite, S. \& Bromfield, C. (2002). Promoting creative thinking through the use of ICT. Journal of Computer Assisted Learning, 18(3), 367.

Yáñez-Luna, J. \& Arias-Oliva, M. (2018). M-learning: aceptación tecnológica de dispositivos móviles en la formación online. Tecnología, ciencia y educación, 10, 13-34. 